\title{
SOBRE O PARADIGMA COGNITIVO NA LINGUUISTICA
}

\author{
Elena Godoy \\ UNICAMP
}

\section{RESUMO}

As pesquisas sobre os fenómenos cognitivos mostram uma forte tendència para a interdisciplinaridade, envolvendo a psicologia, a sociologia, as neurociências, a informática, a lógica, a filosofia e a linguística. Os progressos desses estudos são lentos, os obstáculos consideráveis, os resultados frágeis.

O presente trabalho procura apresentar um panorama dos diferentes programas de investigação cognitiva surgidos na linguística nos últimos anos.

Neste trabalho tentaremos apresentar os contornos gerais do assim chamado paradigma cognitivo do estudo da estrutura gramatical e do funcionamento da linguagem, i.e., daquele conjunto de teorias e programas de pesquisa lingüistica, em que a linguagem é vista como um componente orgânico da mente humana, intimamente relacionado com outros processos e estruturas cognitivos, como percepção, pensamento, atenção, memória, etc.

Muitas das teorias gramaticais modernas impregam-se cada vez mais de conteúdo cognitivo. Entretanto, as concepções linguiisticas cognitivamente orientadas não são unânimes em relação ao papel e ao lugar da gramática no conjunto de estruturas e processos cognitivos. As abordagens cognitivas são diretamente ligadas às tentativas de uma revisão radical de algumas teorias científicas. A orientação cognitivista das pesquisas cientificas que começara na psicologia, linguiística e inteligência artificial está alcançando a sociologia (Cicourel, 1974), a crítica literária (De Beaugrande, 1983), a informática (Ingwersen, 1984), a neuropsicologia (Allport, 1983), a biologia (Nellgren, 1983, etc. 
De acordo com de MEY (1982), duas direçōes na reorientação das pesquisas foram de maior importância para a formação da abordagem cognitivista:

1 - o deslocamento da atenção dos pesquisadores do objeto da cognição para o sujeito desta cognição. (Este processo pode ser visto como substituiçāo do paradigma cartesiano, que entende a razão como estática e passiva, pelo paradigma hegeliano, que vê a razão como ativa nos processos da aquisição de conhecimento);

2 - o deslocamento da atenção dos pesquisadores das microunidades estudadas isoladamente para as unidades de alto grau de complexidade (até os macromodelos do universo). Na lingiiistica, a primeira direção funciona, por exemplo, quando a compreensão da fala é vista como atividade construtiva do sujeito, realizada na base do conhecimento que ele possui; a segunda direçāo revela-se, por exemplo, quando para a análise da compreensāo dos enunciados relativamente simples, são chamadas as unidades razoavelmente grandes. Em princípio, toda pesquisa orientada para o estudo dos aspectos cognitivos da estrutura e do funcionamento da linguagem pode ser chamada de lingüístico-cognitiva. S.M.LAM (1984) considera a linguiística cognitiva ser uma área da lingüistica, reconhecendo, entretanto, sua estreitissima ligação com a psicologia cognitiva, a antropologia cogtiva e a inteligência artificial. $\mathrm{Na}$ verdade, os pesquisadores da inteligência artificial dão muito mais atenção à compreensão dos enunciados lingüísticos, aos processos e estruturas que servem de base para esta compreensão do que os próprios linguiistas.

A linguística cognitiva caracteriza-se por uma grande variedade de direçōes e programas de pesquisa. Lembremos, por exemplo, a psicossemântica de W. Chafe, a psicolexicologia de G.A.Miller e P.N. Johnson-Laird, a semântica cogniti. va e a teoria cognitiva do uso da linguagem de $T$. van Dijk, a lingüística experiencial de $G$. Lakoff, etc. As várias abordagens existentes se distinguem pela sua orientação teórica e também em relação aos objetivos, métodos e dados empíricos escolhidos. Esta situação, bastante típica das pesquisas interdiciplinares, é determinada pelo fato de que as abordagens linguístico-cognitivas mantêm certa semelhança de familia com as suas fontes: psicologia cognitiva, inteligência artificial, lógica formal, psicolingüística, gramática funcional, gramática gerativa, pragmática, etc. Assim, por exemplo, na linguística cognitiva de R.Fawcett dominam claramente as características da linguística funcional, na abordagem de R. Jackendoff, as da gramática gerativa, na semântica cognitiva de T. van Dijk, as da psicologia cognitiva. 
Um dos fatores integrantes da linguística cognitiva é a exigência da realidade psicológica colocada em relaçāo a qualquer teoria orientada para o estudo da linguagem e seu funcionamento. A revelação da realidade psicológica da teoria em questão transforma-se em uma espécie de imperativo categórico que pode ser seguido, entretanto, de maneiras diferentes. J.GOODWIN e U.HEIN (1982) destacam duas posições principais que podem ser tomadas em relação à realidade psicológica: a forte e a fraca. Os partidários da posição forte (G.MILLER, P.JOHNSON-LAIRD, R.SCHANK e outros) consideram como psicologicamente real aquela teoria do funcionamento da linguagem que ao mesmo tempo é a teoria do funcionamento da mente humana. Uma das representantes da posição fraca é a chamada posição oportunista que se caracteriza pelo uso do seguinte princípio: "O modelo . . . não é uma teoria psicológica de um fenômeno dado. mas sua explicação psicológica deve se basear neste modelo" (J. GOODWIN; U. HEIN; 1982, p. 270). Os autores afirmam que justamente este principio foi usado por N. Chomsky em Aspects. . Esta posição é cômoda por ser invulnerável: quaisquer argumentos contra a inconsistência psicológica de algum dos seus postulados são rebatidos, alegando-se o caráter puramente descritivo do modelo em questão, enquanto cada face psicologicamente atraente do modelo é apresentada de modo mais vantajoso.

De qualquer maneira, a inclusão das teorias da linguagem na teoria geral do conhecimento é reconhecida atualmente como de maior importância. Com isso, o papel da semântica, como um elo que une a teoria linguística com as teorias sobre outras faculdades cognitivas, torna-se essencial, porque "estudando a semântica da língua natural, nós necessariamente estudamos a estrutura do pensamento" ( $R$. JACKENDOFF; 1983, p.X). Em alguns trabalhos, (R. JACKENDOFF, 1983; G. LAKOFF, 1982; P. JOHNSON-LAIRD, 1982; G.MILLER, 1978), é defendido o ponto de vista, conforme o qual, a estrutura semântica, i.e., aquela informação que é transmitida por meio da linguagem, é predeterminada pela maneira de como é organizada a experiência pela mente humana.

Com base nesta concepção, JACKENDOFF propõe encarar a semântica da língua natural como um componente da teoria geral da estrutura conceitual e apresenta uma restrição cognitiva sobre a teoria semântica, de acordo com a qual é postulada a existência dos niveis da representação mental, onde a informação transmitida através dos meios linguiisticos poderia ser confrontada com a informaçāo oferecida pela visão, percepção auditiva não verbal, olfato, sis- 
tema sensório-motor, etc. O autor afirma que esta restrição pode ser útil para o estudo dos meios da reflexão da natureza do pensamento pela forma sintática da lingua.

Evidentemente, a grande atençāo dada na teoria cognitiva da linguagem aos vários componentes da mente humana leva à reavaliação do papel da gramática. Assim, para $H$. CLARK e S.HAVILAND (1974), a tarefa central da abordagem cognitiva do estudo da linguagem é a especificação dos processos de compreensão e de produção dos enunciados. Portanto, nesta concepção, o conhecimento da gramática tor. na-se um criado e não um dono destes processos. A posição semelhante é a de R.FAWCETT (1980) que considera a gramática (ou até todo o sistema lingüístico) como apenas um dos componentes do modelo cognitivo da mente interativa pela sua natureza. No seu modelo FAWCETT inclui também:

- o registrador de necessidades (fisiológicas e psicológicas;

- o solucionador de problemas, que elabora os planos da solução dos problemas condicionados pelas necessidades fisiológicas ou psicológicas;

- o conhecimento do mundo (as informações gerais são guardadas na memória remota e as informaçôes concretas, relevantes para o ato de fala dado, na memória operativa);

- os estados emocionais;

- os códigos semióticos não verbais;

- os programas de construção de texto;

- os programas comportamentais não comunicativos.

Para T.WINOGRAD (1972), o paradigma cognitivo do estudo da linguagem deve se basear nos seguintes principios fundamentais:

1 - a estrutura do conhecimento do falante é a área mais importante da pesquisa;

2 - este conhecimento pode ser entendido como um conjunto de regras formais que pertencem às estruturas de simbolos.

$\mathrm{Na}$ sua teoria da linguagem, uma atenção especial é dada ao dinamismo dos processos cognitivos e ao conhecimento processual da semântica:

- No núcleo da teoria da linguagem estão os mecanismos básicos da produção e compreensão dos enunciados nos contextos linguístico e pragmático.

- As propriedades principais da fala refletem a estrutura mental do falante, incluindo os minimos detalhes da memória, os algoritmos do processamento de conhecimento e a limitação inata das faculdades de cálculo. 
- A produção e a compreensāo de enunciados acontecem dentro dos limites da estrutura dos processos da atividade mental que incluem elementos linguísticos e extralinguiísticos. Alguns enunciados só podem ser entendidos quando se leva em conta o papel que eles desempenham neste contexto mais amplo.

- Todo enunciado é produzido para realizar uma certa combinação de objetivos comunicativos através dos elementos e seleções oferecidos pela língua. O significado é multidimensional e é formalizável só em termos de todo o conjunto de objetivos e conhecimentos, dos quais o falante e o ouvinte dispöem.

- Os formalismos mais adequados para a construção das teorias do pensamento e da linguagem devem ser explicitamente ligados à estrutura do conhecimento, tal como este é guardado no sistema dos processos mentais, e à estrutura dos processos que se servem deste conhecimento.

- As estruturas de símbolos e os processos que as operam desempenham o papel principal na formalizaçāo da atividade mental. Não existe uma estrutura estática única que possa representar o significado do enunciado. Na realidade, existe um conjunto de estruturas construídas e modificadas pelo falante e pelo ouvinte no ato de fala, e a teoria deve explicar a sequiência de estruturas e a natureza de modificações.

$E$ interessante notar que as pesquisas dentro do paradigma cognitivo inclinam-se cada vez mais para o estudo do conjunto dos conhecimentos usados pelo homem. De acordo com M. de MEY (1982), por exemplo, as ciências cognitivas estudam a natureza do conhecimento e os meios possiveis de sua transformação. A psicologia cognitiva que surgiu nos anos 60 tem como objetivo "o estudo do conhecimento e dos meios de sua representação" (GELDER, 1982). O conhecimento desempenha o papel principal também nos sistemas de inteligência artificial. $O$ próprio conceito de inteligência é relacionado freqüentemente com a capacidade de usar "o conhecimento necessário no momento necessário" (BOBROW, 1975). Na linglística a situaçāo é semelhante. Por exemplo, W.CHAFE (1972) afirma que "se nós realmente almejamos compreender o funcionamento da linguagem, não temos outra alternativa do que atacar de frente o problema de como o nosso conhecimento do mundo influencia aquilo que nós dizemos sobre o mudo". $R$. JACKENDOFF considera como tarefa principal da teoria linguística a descrição "daquilo que o homem sabe quando sabe como dizer" (JACKENDOFF, 1978). 
Alguns lingüistas entendem a própria linguagem como um processo cognitivo baseado no conhecimento. Em todo caso, o estudo da linguagem é aceito como um dos caminhos possiveis para o estudo da natureza do conhecimento (CHOMSKY, 1982).

Entre os resultados mais importantes do estudo do conhecimento necessário para a compreensão e produção da linguagem podemos apontar:

- a constatação da enorme complexidade dso processos cognitivos que fundamentam até os atos de fala mais elementares:;

- o reconhecimento do fato de que o conhecimento propriamente lingüístico é insuficiente para a compreensão de enunciados;

- a consciência de que "as teorias da linguagem devem ser explicitamente teorias da formalização e organização do conhecimento" (WINOGRAD, 1972, p. 390).

Podemos dizer que a abordagem cognitiva supõe o estudo do conhecimento como um iceberg: o conhecimento revelado, expressado explicitamente representa apenas uma pequena parte do conhecimento realmente acionado (de MEY, 1982). A base cognitiva usada nos processos do funcionamento linguístico no é um depósito de informação estático. Na opinião de T.MOORE e Ch.CARLING (1982, p. 11), esta base "é antes um sistema auto-organizador e auto-regula. dor, móvel e modificado na base de novos dados. Por isso a compreensão de qualquer fragmento de Pala exige que o indivíduo use a base de dados que se adapta constantemente. A compreensão de qualquer enunciado leva por sua vez à reorganização subseqüente desta base de dados". Esta base do conhecimento deve incluir, ao menos, os seguintes componentes:

1 - Conhecimento linguístico:

- o conhecimento da língua, i.e., da sua gramática (junto com a fonética e a fonologia) e da sua semântica (lexical);

- o conhecimento do uso da língua, i.e., do uso dos registros, dos dêiticos, etc.;

- o conhecimento dos princípios pragmáticos, necessário para expressar e interpretar a referência, para expressar e compreender a polidez, a ironia, o sarcasmo, para interpretar os atos ilocutórios, etc.

2 - Conhecimento extralinguístico:

- sobre o contexto e a consituação da enunciação, i.e., sobre os objetos e suas propriedades funcionais, sobre 0 ouvinte, incluindo o conhecimento de seus objetivos e pla- 
nos, das imagens que ele tem sobre o falante e sobre o mundo, etc.;

- o conhecimento do mundo.

Como se sabe, na linguística durante muito tempo existiu uma reserva (se não o desprezo) em relação ao conhecimento extralinguiistico. É curioso observar a atitude semelhante em relação ao conhecimento linguístico existente entre os estudiosos da inteligência artificial. R. SCHANK (1982; 1984), por exemplo, afirma que "as regras gramaticais não são decisivas para a compreensão" e que as decisivas mesmo são as "regras" da realidade".

M. DASCAL (1981) aponta para pelo menos quatro pontos de vista em relação ao contexto: contextualismo radical, contextualismo moderado, "literalismo" moderado e "literalismo" radical. O contextualismo moderado procura explicar a maior parte do conteúdo semântico da sentença/enunciado pela ação dos fatores contextuais. O contextualismo radical (J. SEARLE, 1983; 1984) nega a existência de qualquer significado invariante (livre do contexto) da sentença. Ao contrário, o "literalismo" radical não reconhece a possibilidade de quaisquer componentes do significado da sentença serem predeterminados pelos fatores contextuais. Na visão do "literalismo" moderado, por menor que seja o papel do significado literal da sentença, ele deve ser levado em conta. A contribuição do significado literal é mais no sentido de identificar os elementos do conhecimento do mundo relevantes para o enunciado em questão.

Parece evidente que o que está à disposição dos participantes de um ato de fala nāo são as situaçōes, o ambiente ou os fragmentos anteriores do discurso, mas antes o conhecimento e as representações destes elementos. Disso segue que o contexto deve ser definido como o conjunto de representações (BROCKWAY, 1981). Contudo, parece pouco provável que no processo de fala seja usado todo o conjunto de conhecimentos e representações que os falantes possuem. Por isso torna-se necessária a elaboração de um critério para a restrição dos fatores que entram na interpretação do enunciado.

Em alguns trabalhos (SMITH, 1982, por exemplo), aparece a convicçāo de que o contexto pode ser reduzido aos conhecimentos, representaçōes e pressuposiçōes comuns aos participantes de um ato de fala. De acordo com este ponto de vista, o conhecimento do mundo é a premissa principal para a compreensão de fala.

D.SPERBER e D.WILSON (1982), ao contrário, afirmam que relacionar uma proposição com o fundo do conhecimento geral é, na verdade, o resultado da compreensão e não sua 
premissa. Com isso, os autores frisam a natureza dinâmica do contexto.

De acordo com M.STEEDMAN e P.JOHNSON-LAIRD (1980), a modelação dinâmica dos conhecimentos e objetivos do ouvinte tem a importância decisiva para o sucesso do ato de fala. $E$ natural que o falante, modelando o conhecimento do ouvinte, identifique este conhecimento com seu próprio (quando não há indícios de que esta identificação seja infundamentada).

A orientação para o conhecimento do ouvinte é um dos principios organizadores da atividade de fala. D.SPERBER e D. WILSON (1982) chamam este principio de princípio de relevância.

A importância do papel do conhecimento do mundo recebeu uma expressão explicita na hipótese de relatividade cognitiva de M.KRRECKEL (1981). Esta hipótese baseia-se no postulado, de acordo com o qual, "o grau, de compreensão alcançada no processo de fala é diretamente proporcional ao grau de convergência entre os participantes do ato de fala no domínio dos conceitos internacionalmente relevantes $e$ dos meios convencionais de sua expressão" (KRECKEL, 1981, p. 4). A este postulado subordinam-se os seguintes:

- A organização conceitual da realidade realizada pelo individuo tem o caráter único. Esta organizaçāo reflete sua experiência de interação com o mundo e seu conhecimento do mundo.

- Os conceitos, dos quais o indivíduo dispõe, são organizados de maneiras diferentes e expressados de modos diferentes, dependendo do sistema semiótico (subcódigo), ao qual pertencem. Cada indivíduo dispōe de um determinado repertório de subcódigos.

- A organização conceitual da realidade e os meios de expressão dos conceitos têm o caráter dinâmico (e não estático).

- A organização conceitual determina os meios de expressão lingüística.

- A interação realizada numa perspectiva comum para os participantes do ato de fala reforça a convergência dos conceitos e meios linguísticos usados.

- A interação realizada no passado com a perspectiva geral da possibilidade da interação no futuro contribui para a formação de um subcódigo específico.

- A probabilidade da realização ótima da comunicação é máxima se os seus participantes utilizam o mesmo subcódigo baseado no volume considerável do conhecimento comum. 
Um dos resultados importantes produzidos pelo paradigma cognitivo é a noção da interrelação dos processos que se realizam na memória humana e os processos que determinam a produção e a compreensão dos enunciados. R.SCHANK frisa que "devemos estar conscientes de que qualquer teoria do processamento da lingua natural deve ser também a teoria da memória" (SCHANK, 1982, p. 457).

Muitos cognitivistas dão uma atençāo especial à organização dos resultados da atividade cognitiva na memória remota e principalmente à organização categorial e à formação de estruturas de alto nivel de representaçāo de experiência passada. Para a lingüistica cognitiva, a importância do estudo da organização categorial da memória remota é determinada pelo fato de que a língua natural só permite comunicar algo em termos categoriais.

De acordo com G.LAKOFF (1982), a recente revolução na psicologia cognitiva é relacionada com a mudança do conceito de categoria. Foi descoberto que o homem classifica as coisas de maneira bem diferente do que isso é feito nos modelos linguiisticos. Conforme a hipótese de E.ROSCH (1978), as particularidades da organização categorial não podem ser vistas como arbitrárias e historicamenie acidentais. Ao contrário, estas particularidades são resultado da ação de certos principios psicológicos universais. EROSCH distingue dois princípios: "o principio da economia cognitiva" e "o princípio da estrutura do mundo percebida". O primeiro principio tem a ver com o funcionamento dos sistemas categoriais. De acordo com este principio, a tarefa dos sistemas categoriais é fornecer o máximo de informação com gastos cognitivos mínimos. Duas tendências contrárias entram em jogo na categorização: 1) a diferenciação máxima das categorias, e 2) a limitação da diferenciaçāo. Conforme - segundo princípio da categorização, o mundo não pode ser visto como um conjunto desestruturado de traços coocorrentes equiprováveis. Por isso o máximo de informação com os gastos coginitivos mínimos será fornecido pelo sistema categorial que reflete com maior exatidão a estrutura do mundo percebido.

De acordo com hipotese de E.ROSCH, as categorias são determinadas pelas propriedades (perceptivas, funcionais, etc.) dos objetos prototípicos destas categorias. Os postulados principais da abordagem prototípica são os seguintes:

- Central tendency. Uma categoria não pode ser definida nem como um conjunto, nem como uma intersecção de traços dos seus componentes. Antes, ela pode ser definida em termos de prototipicidade ou centralidade (central 
tendency) do elemetno que tem o número maximo de traços comuns com os outros elementos do conjunto.

- Semelhança de família. Se um elemento pertence ou não a uma categoria dada é determinado na base de sua semelhança de familia com o protótipo.

- Goodness of category membership: é determinada pela quantidade dos traços comuns com o protótipo.

- Heterogeneidade das categorias (heterogeneous membership). Dois elementos podem pertencer a uma mesma categoria, quando têm os traços comuns com o protótipo, mesmo nāo havendo os traços comuns para estes dois elementos.

- Fuzziness: os limites das categorias não são bem definidos e podem deslocar-se de acordo com as necessidades do indivíduo.

- Weighting: alguns traços do protótipo têm o peso maior que os outros.

E.BATES e B.MACWHINNEY (1982), também supõem que as categorias gramaticais sāo organizadas em torno de elementos prototípicos. Na sua opinião, o modelo prototípico pode ser usado para descrever a interação dos fatores semânticos e pragmáticos, como, por exemplo, o agente e o tópico - as categorias que estão na base da categoria de sujeito.

A importância fundamental da experiência passada na memorização e compreensão de enunciados foi demonstrada pela primeira vez por F.BARLETT (1932) que descobriu que a memória quase nunca é literal. Na reprodução de um texto memorizado, 0 indivíduo frequientemente $o$ modifica de acordo com os estereótipos e normas cognitivos existentes no seu meio social. Para descrever a representação da informaçăo na memória, F.BARLETT usou o conceito de frame que ele entendia como a organização ativa da expeirência passada. Sua teoria parte do pressuposto de que a compreensão é determinada pela descoberta do frame guardado na memória. B.KUIPERS (1975) usa a teoria de frames de representação do conhecimento para explicar como a organização da experiência anterior na memória influencia a percepção ativa e a compreensão.

As estruturas de conhecimento chamadas frames são, na verdade, os pacotes de informação (guardados na memória ou criados nela - em caso de necessidade - dos componentes guardados na memória) que garantem o processamento cognitivo satisfatório das situaçōes I.STEELS (1981) apresenta os seguintes princípios da formação dessas estruturas: 
- os conhecimentos relevantes para um certo dominio são agrupados juntos;

- os conhecimentos de um domínio são organizados dos elemetnos que refletem a estrutura conceitual deste dominio;

- as unidades da informação que compōem uma estrutura são ordenadas.

D.METZING (1981), simplificando o esquema acina, afirma que as "representações de frame" são "pedaços do conhecimento" recortados de maneira ótima para serem usados no processamento da informação.

Finalizando a apresentação do levantamento bibliográfico da literatura dedicada ao paradigma cognitivo, reconhecemos que este levantamento não é completo rem exaur: tivo. No entanto, acreditamos que ele poderá ser útil para os linguiistas, psicólogos e especialistas em inteligência artificial.

\section{BIBLIOGRAFIA}

ALLPORT, D. Language and cognition. In: Approaches to language. Oxford, 1983. p.61-94.

BARLETT, F. Remembering: A study in experimental and social psy. cology. Cambridge, 1932.

BATES, E.; B. MacWHINNEY. Funcionalist approaches to grammar. In: Language acquisition: The state of the art. Cambridge, 1982. p.173.218.

BEAUGRANDE, R. de. Surprised by syncretism. Poetics, v.12, n. 2/3, p.83-137, 1983.

BOBROW, D. Dimensions of representation. In: Representation and understanding. New York, 1975. p.1-34.

BROCKWAY, D. Semantic constraints on relevance. In: Possibilities and limitations of pragmatics. Amsterdam, 1981. p.57-78.

CHAFE, W. Language and memory. Language, v.49, n.2, p.261-281, 1973. CHOMSKY, N. On the generative enterprise. Dordrecht, 1982.

CICOUREL, A. Cognitive sociology: Language and meaning in social interaction. New York, 1974.

CLARK, H.; S. HAVILAND. Psychological processes as linguistic explanation. In: Explaining linguistic phenomena. New York, 1974. p.91124.

DASCAL, M. Contextualism. In: H. PARRET et al. (eds.) Possibilities and limitations of pragmatics. Amsterdam, 1981. p.153-177.

ge I. Amsterdam, 1983.

DIJK, T. van. Dialogue and cognition. In: Cognitive constraints on communication: Representations and processes. Dordrecht, 1984. 
FAWCETT, R. Cognitive linguistics and social interaction. Heideiberg, 1980.

GELDER, B. (ed.) Knowledge and representation. London, 1982. p.1-15. GOODWIN, J. \& U.HEIN. Artificial intelligence and the study of lan. guage. Journal of pragmatics, v. 6, n. 2, p. 241-280, 1982.

INGWERSEN, P. Psychological aspects of information retrieval. Social science and information studies. v.4, n.2/3, p.83-95, 1984.

JACKENDOFF, R. Grammar as evidence for conceptual structure. In: Linguistic theory and psychological reality. Cambridge, 1978. p. 201-228.

- Semantics and cognition. Cambridge, 1983.

JOHNSON-LAIRD, P. Formal semantics and the psychology of meaning. In: Processes, beliefs and questions. Dordrecht, 1982. p. 1-68

- Mental models: Toward a cognitive science of language, inference and consciousness. Cambridge, 1983.

KRECKEL, M. Communicative acts and shared knowledge in natural discourse. London, 1981.

KUIPERS, B. A frame for frames: Representing knowledge for recognition. In: Representation and understanding. New York, 1975. p. 151-183.

LAKOFF, G. Experiential factors in linguistics. In: Language, mind, and brain. London, 1982, p.145-156.

LAM, S. On the aims of linguistics. In: New directions in linguistics and semiotics. Amsterdam, 1984. p.1-11.

MELLGREN, R. (ed.) Animal cognition and hehaviour. Amsterdam, 1983.

METZING, D. Frame representation and lexical semantics. In: Words, worlds, and contexts: New approaches in word semantics. Berlin/ New York, 1981, p. 320-342.

MEY, M. de. The cognitive paradigme. Dordrecht, 1982.

MILLER, G. Practical and lexical knowledge. In: Cognition and categorization. Hillsdale, 1978, p.305-319.

MOORE, T.; Ch. CARLING. Understanding language. London, 1982.

ROSCH, E. Principles of categorization. In: Cognition and categorization. Hillsdale, 1978. p. 27-48.

SCHANK, $R$. Depths of knowledge. In: Knowledge and representation. London, 1982. p.170-193.

Looking for a process model of dialogue: Speculations from the perspective of artificial intelligence. In: Cognitive constraints on communication: Representations and processes. Dordrecht, 1984. p. $161 \cdot 173$.

- Reminding and memory organization. In: Strategies for natural language processing. Hillsdale, 1982. p.455-493.

SEARLE, J. Intentionaly: An Essay in the Philosophy of Mind, Cambridge, 1983 .

Minds, Brains and Science. Cambridge, 1984. 
SMITH, N. (ed.) Mutual knowledge. N.Y./London, 1982. p.61-87. SPERBER, D. \& D. WILSON. Mutual knowledge and relevance in theories of comprehension. In: Mutual knowledge. N.Y./London. 1982. p.61-87.

STEEDMAN, M. \& P. JOHNSON-LAIRD. The production of sentences utterances and speech acts: Have computers anything to say? In: Language production. London, v.1, p.111-142, 1980.

STEEIS, L. Frames and descriptions. In: Emplrical semantics. Bochum, v.1, p.192-235, 1981.

WINOGRAD, T. Understanding natural language. New York, 1972. 